\title{
AN APPROXIMATE STOCHASTIC MODEL FOR PHAGE REPRODUGTION IN A BACTERIUM
}

\author{
J. GANI \\ (received 27 July 1961, revised 18 October 1961)
}

\section{Introduction}

The stochastic birth-death process considered in this paper provides an approximate model for phage reproduction in a bacterium. In a recent paper, Hershey [1] has discussed reproduction and recombination in phage crosses, and a deterministic model for the reproductive process has been the subject of a previous note by the author [2]. A very readable account of the process is given by Sanders [3] in his recent article, "The life of viruses".

Consider a medium initially containing $N<\infty$ bacteria, which we may for simplicity assume to reproduce as a birth process with constant parameter. Into this medium is inserted one phage particle (or several), which immediately penetrates (infects) a bacterium, and proceeds to reproduce within it as a birth-death process with constant parameters $\lambda>\mu$. After a phage has invaded a bacterium, changes occur on the surface of the bacterium to prevent its penetration by further phages. Usually a single phage invades a bacterium, but towards the end of the infective process when the number of phages and bacteria are of the same order, there is a larger probability that a bacterium is infected by two or more phages simultaneously; in our model, this probability is taken to be negligible. The death of a phage corresponds in fact to its reaching maturity, after which it no longer reproduces. When a fixed number $r$ (two to three hundred) of these mature phages have been produced, the bacterium, which is itself incapable of fission after infection by a phage, breaks open as it dies, releasing the phage offspring. Immature phages cannot attack bacteria, but the $r$ mature phages immediately penetrate a further $r$ uninfected bacteria; the phages reproduce faster than the bacteria, and this sequence of processes usually continues until the bacteria are all dead.

Let us now discuss the phage reproduction occurring in a single bacterium. We should like to find the distribution of time $T_{r}$ up to the occurrence of the $r$-th death in an ordinary birth-death process: this is not in fact known. Assuming for the moment that the process continues indefinitely, without 
stopping when $r$ mature phages are produced, it is possible to write the differential equations for the probabilities.

$$
P_{i j}(t)=\operatorname{Pr}\{i \text { survivals and } j \text { deaths in time } t\}
$$

in the form

$$
P_{i j}^{\prime}(t)=(i-1) \lambda P_{i-1, i}(t) \rightarrow i(\lambda+\mu) P_{i j}(t)+(i+1) \mu P_{i+1, j-1}(t)
$$

for all values of $i, j=0,1,2, \cdots$, with $P_{i j}(t)$ identically zero for $i=j=0$, and $i$ or $j<0$.

If we further define the joint probability generating function (p.g.f.) $\phi(u, v, t)$ as

$$
\phi(u, v, t)=\sum_{i, j=0}^{\infty} P_{i j}(t) u^{i} v^{j} \quad(|u|,|v| \leqq 1),
$$

the equations (1.1) lead to the partial differential equation

$$
\frac{\partial \phi}{\partial t}=\left\{\lambda u^{2}-(\lambda+\mu) u+\mu v\right\} \frac{\partial \phi}{\partial u},
$$

which, when $v=1$, reduces to the well-known equation for the generating function of probabilities of survivals. The equation (1.3) can be solved: solutions have in fact been obtained for somewhat different but equivalent forms of it by Kendall [4] and Bartlett [5]. However, expansion of the p.g.f. is unwieldy, and it seems difficult to obtain the $P_{i j}(t)$ explicitly from it. A different approach using the Laplace transforms with respect to time

$$
q_{i j}(s)=\int_{0}^{\infty} e^{-s t} P_{i j}(t) d t \quad(R(s)>0)
$$

leads to the relations

$$
\{(\lambda+\mu)+s\} q_{10}(s)=1 \text { for } i=1, j=0,
$$

$\{i(\lambda+\mu)+s\} q_{i j}(s)=(i-1) \lambda q_{i-1, j}(s)+(i+1) \mu q_{i+1, j-1}(s)$ for all other $i, j$, with $q_{i j}(s)$ identically zero for $i=j=0$, and $i$ or $j<0$. These are again not readily solved.

\section{An approximation to the birth-death process}

It is natural at this point to approximate to the standard birth-death process by one for which it is possible to obtain the probabilities $P_{i j}(t)$ explicitly. Such a process is that where births occur in a non-homogeneous Poisson process, the probability of a birth in the interval $(t, t+\delta t)$ being

$$
\lambda 2^{\alpha t} \delta t+o(\delta t)
$$


where $\alpha=\lambda-\mu>0$ and $e^{\alpha t}$ is the mean number of survivals at time $t$ in the standard birth-death process. The death process remains unchanged. We should now strictly refer to probabilities $P_{i j}(0, t)$ and the p.g.f., $\phi(u, v ; 0, t)$, since the process is no longer homogeneous in time; for simplicity, however, we retain the notations $P_{z j}(t), \phi(u, v, t)$ which are quite clear in this case.

The forward differential equations for these probabilities are now

$$
P_{i j}^{\prime}(t)=\lambda e^{\alpha t} P_{i-1, j}(t)-\left(\lambda e^{\alpha t}+i \mu\right) P_{i j}(t)+(i+1) \mu P_{i+1, j-1}(t)
$$

for all values of $i, j=0,1,2, \cdots$, with $P_{i j}(t)$ identically zero for $i=j=0$ and $i$ or $j<0$. The joint p.g.f., $\phi(u, v, t)$, satisfies the equation

$$
\frac{\partial \phi}{\partial t}+\mu(u-v) \frac{\partial \phi}{\partial u}=\lambda e^{\alpha t}(u-1) \phi
$$

which we proceed to solve. If we perform the transformation $T=(u-v) e^{-\mu t}$ leaving $u, v$ unchanged, and write

$$
F(u, v, T)=\phi(u, v, t)
$$

we obtain from (2.3) that

$$
\frac{\partial F}{\partial u}=\frac{\lambda}{\mu}(u-1)(u-v)^{(\alpha / \mu)-1} T^{-\alpha / \mu} F(u, v, T) .
$$

The solution to this equation is of the form

$$
\begin{aligned}
F(u, v, T) & =\left\{\exp \frac{\lambda}{\mu} T^{-\alpha / \mu} \int_{u_{\bullet}}^{u}(x-1)(x-v)^{(\alpha / \mu)-1} d x\right\} f(v, T) \\
& =\left\{\exp T^{-\alpha / \mu}\left[(u-v)^{\lambda / \mu}+\frac{\lambda}{\alpha}(v-1)(u-v)^{\alpha / \mu}-A\right]\right\} f(v, T)
\end{aligned}
$$

where $A$ is a function of $v$ which later vanishès. Rewriting this as $\phi(u, v, t)$, we find that

(2.6) $\phi(u, v, t)=\left\{\exp e^{\alpha t}\left[(u-v)+\frac{\lambda}{\alpha}(v-1)-A(u-v)^{-\alpha / \mu}\right]\right\} f\left(v,(u-v) e^{-\mu t}\right)$

The initial condition $P_{10}(0)=1$ results in

$$
\phi(u, v, 0)=u=\left\{\exp \left[(u-v)+\frac{\lambda}{\alpha}(v-1)-A(u-v)^{-\alpha / \mu}\right]\right\} f(v, u-v)
$$

whence it follows that

$$
f(v, T)=(T+v) \exp -\left\{T+\frac{\lambda}{\alpha}(v-1)-A T^{-\alpha / \mu}\right\}
$$


Thus $\phi(u, v, t)$ can finally be written as

$$
\begin{aligned}
\phi(u, v, t) & =\left\{v+(u-v) e^{-\mu t}\right\} \exp \left\{e^{\alpha t}\left[(u-v)+\frac{\lambda}{\alpha}(v-1)-A(u-v)^{-\alpha / \mu}\right]\right. \\
& \left.-\left[(u-v) e^{-\mu t}+\frac{\lambda}{\alpha}(v-1)-A(u-v)^{-\alpha / \mu} e^{\alpha t}\right]\right\} \\
& =\left\{u e^{-\mu t}+v\left(1-e^{-\mu t}\right)\right\} \exp \left\{-\frac{\lambda}{\alpha}\left(e^{\alpha t}-1\right)\right. \\
(2.9) \quad & \left.+u\left(e^{\alpha t}-e^{-\mu t}\right)+v\left[\frac{\lambda}{\alpha}\left(e^{\alpha t}-1\right)-\left(e^{\alpha t}-e^{-\mu t}\right)\right]\right\} \\
& =\left\{u e^{-\mu t}+v\left(1-e^{-\mu t}\right)\right\} \exp \{-\rho(t)+u \Lambda(t)+v[\rho(t)-\Lambda(t)]\} \\
& =e^{-\rho(t)}\left\{\sum_{i=0}^{\infty} \sum_{j=0}^{\infty} u^{i} v^{j+1}\left(1-e^{-\mu t}\right) \frac{\Lambda^{i}}{i !} \frac{(\rho-\Lambda)^{j}}{j !}\right. \\
& \left.+\sum_{i=0}^{\infty} \sum_{j=0}^{\infty} u^{i+1} v^{j} e^{-\mu t} \frac{\Lambda^{i}}{i !} \frac{(\rho-\Lambda)^{j}}{j !}\right\}
\end{aligned}
$$

where for $t>0, \rho(t)=(\lambda / \alpha)\left(e^{\alpha t}-1\right)>0, \Lambda(t)=e^{\alpha t}-e^{-\mu t}=$ $(1+\alpha \rho / \lambda)^{-\mu / \alpha}\left\{(1+\alpha \rho / \lambda)^{\lambda / \alpha}-1\right\}>0$, and it is easily shown that $\rho(t)-\Lambda(t)>0$.

The p.g.f. for the probabilities of survivals, is given by

$$
\phi(u, 1, t)=\left\{u e^{-\mu t}+\left(1-e^{-\mu t}\right)\right\} e^{-\Lambda(t)(1-u)},
$$

while that for the probabilities of deaths is

$$
\phi(1, v, t)=\left\{v\left(1-e^{-\mu t}\right)+e^{-\mu t}\right\} e^{-\{\rho(t)-\Lambda(t)\}(1-v) .}
$$

\section{Explicit probabilities of births, survivals and deaths}

For the birth-death process continuing indefinitely, without stopping when $r$ mature phages have been produced, it follows from (2.9) directly that the probabilities $P_{i j}(t)$ are

$$
P_{0 j}(t)=e^{-\rho(t)}\left(1-e^{-\mu t}\right) \frac{\{\rho(t)-\Lambda(t)\}^{j-1}}{(j-1) !} \quad(j \geqq 1)
$$

(3.1) $P_{i 0}(t)=e^{-\rho(t)-\mu t} \frac{\{\Lambda(t)\}^{i-1}}{(i-1) !}$

$$
(i \geqq 1)
$$

$$
P_{i j}(t)=e^{-\rho(t)}\left\{\left(1-e^{-\mu t}\right) \frac{\Lambda^{i}}{i !} \frac{(\rho-\Lambda)^{j-1}}{(j-1) !}+e^{-\mu t} \frac{\Lambda^{i-1}}{(i-1) !} \frac{(\rho-\Lambda)^{j}}{j !}\right\}(i, j \geqq 1)
$$


Clearly the probability $B_{k}(t)$ of $k$ births in time $t$ is of the Poisson form

$$
\begin{aligned}
B_{k}(t) & =\exp \left\{-\int_{0}^{t} \lambda e^{\alpha \tau} d \tau\right\}\left\{\int_{0}^{t} \lambda e^{\alpha \tau} d \tau\right\}^{k} / k ! \\
& =e^{-\rho(t)}\{\rho(t)\}^{k} / k ! \quad(k \geqq 0),
\end{aligned}
$$

the mean number of births in time $t$ being $\rho(t)$. From (2.10), the probabilities $S_{m}(t)$ of $m$ survivals after time $t$ are

$$
\begin{aligned}
S_{0}(t) & =\left(1-e^{-\mu t}\right) e^{-\Lambda(t)} \\
S_{m}(t) & =e^{-\Lambda(t)}\left\{e^{-\mu t} \frac{\Lambda^{m-1}}{(m-1) !}+\left(1-e^{-\mu t}\right) \frac{A^{m}}{m !}\right\}(m \geqq 1)
\end{aligned}
$$

It should be noted that the mean number of survivals after time $t$ for this process is

$$
\frac{\partial}{\partial u} \phi(1,1, t)=e^{\alpha t}
$$

exactly as for the standard birth-death process, while its variance $e^{\alpha t}\left(1-e^{-(\lambda+\mu) t}\right)$ is less than the variance $(\lambda+\mu)(\lambda-\mu)^{-1} e^{\alpha t}\left(e^{\alpha t}-1\right)$ of the standard process. Similarly from $(2.11)$ the probabilities $D_{r}(t)$ of $r$ deaths in time $t$ are

$$
D_{0}(t)=e^{-\mu t-\rho(t)+\Lambda(t)}
$$

$$
D_{r}(t)=e^{-\{\rho(t)-\Lambda(t)\}}\left\{e^{-\mu t} \frac{(\rho-\Lambda)^{r}}{r !}+\left(1-e^{-\mu t}\right) \frac{(\rho-\Lambda)^{r-1}}{(r-1) !}\right\}(r \geqq 1),
$$

and the mean number of deaths in time $t$ is

$$
\frac{\partial}{\partial v} \phi(1,1, t)=1-e^{\alpha t}+\rho(t)=\frac{\mu}{\alpha}\left(e^{\alpha t}-1\right) .
$$

Suppose the process now stops at the $r$-th death, the probability distribution of this death (or maturing of the $r$-th phage), which is improper since there is a non-zero probability that the process ends before, is given by

$$
\begin{aligned}
g(t) d t & =\sum_{i=1}^{\infty} P_{i, r-1}(t) i \mu d t \\
& =\left\{\text { Term in } v^{r-1} \text { of } \frac{\partial}{\partial u} \phi(1, v, t)\right\} \mu d t \\
& =\left\{\text { Term in } v^{r-1} \text { of }\left[e^{-\mu t}(1+\Lambda)+\Lambda v\left(1-e^{-\mu t}\right)\right] e^{-(\rho-\Lambda)(1-v)}\right\} \mu d t \\
& =e^{-(\rho-\Lambda)}\left\{e^{-\mu t}(1+\Lambda) \frac{(\rho-\Lambda)^{r-1}}{(r-1) !}+\Lambda\left(1-e^{-\mu t}\right) \frac{(\rho-\Lambda)^{r-2}}{(r-2) !}\right\} \mu d t
\end{aligned}
$$

We have thus constructed a birth-death process approximating to the 
original one, but for which each of the probabilities of births, deaths and survivals is explicitly known.

If the number of bacteria $N$ is taken to be infinite, an equation for the distribution of the number infected up to time $t$ (or the number of mature phages released up to $t$ ) can be obtained from Bellman and Harris' [6] theory of branching processes. If $Q_{n}(t)$ is the probability of $n$ infected bacteria, and $\psi(s, t)=\sum_{n=1}^{\infty} Q_{n}(t) s^{n}$ the p.g.f. of this distribution, this satisfies the integral equation

$$
\psi\{s, t)=s(1-G(t))+\int_{0}^{t}\{\psi(s, t-\tau)\}^{r} g(\tau) d \tau
$$

where $g(t) d t$ is the improper probability distribution (3.7) and $G(t)$ the function $G(t)=\int_{0}^{t} g(\tau) d \tau$. There seems to be no simple way of solving this equation.

\section{References}

[1] Hershey, A. D., The production of recombinants in phage crosses. Cold Spring Harbor Symposia on Quantitative Biology 23 (1958), $19-46$.

[2] Gani, J., A simple population model for phage reproduction. Bull. Math. Statist. (to appear).

[3] Sanders, F. K., The life of viruses. Penguin Science Survey 1961, 2, 147-159.

[4] Kendall, D. G., On the generalized 'birth-and-death' process. Ann. Math. Statist. 19 (1948), $1-15$.

[5] Bartlett, M. S., Equations for stochastic path integrals. Proc. Camb. Phil. Soc. 57 (1961), 568-573.

[6] Bellman, R. and Harris, T. On age-dependent binary branching processes. Ann. of Math. 55 (1952), 280-295.

Australian National University

Canberra, A.C.T., Australia. 